\title{
Augmented Responses of Angiotensin I and II in Patients with Ischemic Heart Disease
}

\author{
Relation to Left Ventricular Function
}

\author{
Kenichi SeKIGUCHI, MD, Tsugiyasu KANDA, ${ }^{1} \mathrm{MD}$, Zenpei ONO, MD, \\ Toru TANAKA, MD, Tomoyuki YoKOYAMA, MD, Yasushi AIHARA, MD, \\ Takuji TOYAMA, MD, Tetsuo SAKAMAKI, MD, Isao KOBAYASHI, ${ }^{1} \mathrm{MD}$, \\ Masahiko KURABAYASHI, MD, and Ryozo NAGAI, ${ }^{2} \mathrm{MD}$
}

\section{SUMMARY}

To evaluate the effects of left ventricular (LV) dysfunction upon the sympathetic nervous and renin-aldosterone-angiotensin systems, neurohormonal factors were measured in patients with ischemic heart disease. Eleven patients were divided into two groups by their LV ejection fraction based on previous catheterization; preserved $(\mathrm{EF} \geq 60 \%)$ and impaired ( $\mathrm{EF}<60 \%) \mathrm{LV}$ systolic function groups. They performed supine ergometer exercise and blood samples were drawn at rest and at peak exercise. After dynamic exercise, plasma norepinephrine was significantly $(p<0.05)$ increased in patients with preserved LV function, whereas it was not altered in patients with impaired LV function (norepinephrine $20.8 \pm 20.5$ vs $45.8 \pm 41.9$, respectively). We observed no differences in basal or peak levels of neurohormonal factors, including plasma renin activity, aldosterone, and brain natriuretic peptide (BNP), between the groups. Although the plasma levels of angiotensin I and II were not different in the two groups at rest or at peak exercise, their increasing ratios from rest to peak exercise were significantly higher in patients with impaired LV function compared to those with preserved LV function (angiotensin I $;-18.6 \pm 31.0 \%$ vs $64.8 \pm 66.5 \%, p<0.05$, angiotensin II; $-5.9 \pm 41.2 \%$ vs $60.7 \pm 40.4 \%, p<0.05)$. These results suggest that the increasing ratios of angiotensin I and II are superior to BNP as predictors of LV dysfunction, and that the sympathetic nervous system has already been activated even at rest and did not respond to dynamic exercise in patients with LV dysfunction in ischemic heart disease. (Jpn Heart J 2000; 41: 269-278)

Key words: Ischemic heart disease, Ergometer exercise, Angiotensin I, Angiotensin II, Left ventricular function

From the Second Department of Internal Medicine, ${ }^{1}$ Department of Laboratory Medicine, Gunma University School of Medicine, and ${ }^{2}$ Department of Cardiovascular Medicine, Graduate School of Medicine, University of Tokyo, Tokyo, Japan. Address for correspondence: Tsugiyasu Kanda, MD, Department of Laboratory Medicine, Gunma University School of Medicine, 3-39-22, Showa-machi, Maebashi, Gunma 371-8511, Japan. 
Congestive heart failure (CHF) is characterized by decreased cardiac output and neurohormonal activation including sympathetic nervous system stimulation, activation of the renin - angiotensin - aldosterone system (RAAS), and increased secretion of natriuretic peptides. Natriuretic peptides increase the excretion of body fluid and act as a vasodilator, whereas the former two neurohormonal systems have opposite effects. Norepinephrine increases both arterial and venous blood pressure and activates RAAS. In the RAAS, angiotensin II has the most powerful biologic activities. It acts on the adrenal cortex to release aldosterone, the posterior pituitary to release arginine vasopressin, and resistance arterioles to produce intense vasocontriction. ${ }^{1)}$ Thus, they synergistically act to maintain the blood pressure and increase circulating body fluid which results in an increase in cardiac preload and afterload. Therefore, these neurohormonal factors are known to worsen the condition of $\mathrm{CHF}$ and to be activated in patients with $\mathrm{CHF}^{2)}$ and asymptomatic left ventricular dysfunction. ${ }^{3)}$ In patients with $\mathrm{CHF}$, an activated sympathetic nervous system increases the spillover of norepinephrine. ${ }^{4)}$ Both plasma norepinephrine concentration and plasma renin activity correlate with the clinical severity of CHF, and patients with marked neuroendocrine activation showed a poor prognosis. ${ }^{5)}$ Recent randomized controlled trials (CONSENSUS$\mathrm{I}^{6}{ }^{6}$ SOLVD, ${ }^{7)} \mathrm{V}-\mathrm{HeFT}$ II, ${ }^{8)}$ SAVE, ${ }^{9)}$ PRECISE ${ }^{10)}$ ) proved that the angiotensin converting enzyme (ACE) inhibitors or $\beta$-adrenergic antagonists improve the prognosis of patients with $\mathrm{CHF}$, which suggest that it is important to control the excess activation of neurohormonal systems for the treatment of CHF. Plasma levels of neurohormonal factors are affected by many factors, including body position and exercise. ${ }^{1}$ Dynamic exercise in particular dramatically stimulates neuroendocrine systems and its responses in patients with CHF are different from those in normal subjects. ${ }^{11,12)}$ There are several reports indicating that the neurohormonal responses during dynamic exercise in patients with $\mathrm{CHF}$ are more activated than those of the normal controls. The aim of this study was to examine whether the severity of LV dysfunction may affect the neurohormonal responses after dynamic exercise in patients with ischemic heart disease.

\section{METHODS}

Study Groups: Eleven patients with IHD were evaluated. They were all male and their functional capacity corresponded to New York_Heart Association functional class II or III as shown in Table I. Subjects were divided into two groups, preserved (ejection fraction $(\mathrm{EF} \geq 60 \%)$ and impaired $(\mathrm{EF}<60 \%)$ left ventricular (LV) systolic function, by LVEF based on previously performed cardiac catheterization. $64 \%$ of patients were receiving nitrate, $18 \%$ (2) ACE inhibitor, $45 \%$ (5) calcium antago- 
Table I. Patient Characteristics and Clinical Data

\begin{tabular}{lcc}
\hline & Preserved & Impaired \\
\hline Age (y) & $62.8 \pm 8.0$ & $57.8 \pm 6.9$ \\
NYHA II / III (n) & $5 / 1$ & $3 / 2$ \\
AP / OMI (n) & $4 / 2$ & $1 / 4$ \\
LVEF (\%) & $65.8 \pm 4.2$ & $48.7 \pm 6.5^{*}$ \\
\hline
\end{tabular}

NYHA $=$ New York Heart Association functional classification; AP, angina pectoris; $\mathrm{OMI}=$ old myocardinal infarction; $\mathrm{LVEF}=$ left ventricular ejection fraction; ${ }^{*}, p<0.05$.

nist, and $55 \%$ (6) $\beta$-adrenergic antagonist. $\beta$-Adrenergic antagonists were withdrawn for more than $48 \mathrm{~h}$ before exercise testing. No patients were receiving digitalis or diuretics. Patients with diabetes mellitus were excluded.

Exercise testing: A symptom-limited exercise testing was performed on an upright bicycle ergometer. Workload was started at 25 watts and increased stepwise by 12.5 watts every minute until exhaustion. Heart rate and rhythm were monitored from the ECG. Blood pressure was measured at every minute by standard cuff technique. All patients stopped exercise on presentation of dyspnea or leg fatigue, that is, above 18 on the Borg scale. Measurement of neurohormonal factors: Blood samples were obtained from peripheral vein at rest and at peak exercise. Samples were transferred immediately into ice-cold tubes containing ethylenediamine tetraacetic acid sodium, and centrifuged at $3000 \mathrm{rpm}$ for 10 minutes at $4{ }^{\circ} \mathrm{C}$. Plasma was then frozen at $-80^{\circ} \mathrm{C}$ until assayed. The plasma concentration of norepinephrine was assayed by high-performance liquid chromatography. Plasma renin activity, the plasma level of brain natriuretic peptide (BNP), aldosterone, and angiotensin I and II were determined by radioimmunoassay. The increasing ratios of plasma angiotensin I and II from rest to peak exercise were evaluated as follows. (The increasing ratio of angiotensin $\mathrm{I})=\{($ angiotensin I at peak exercise $)-($ angiotensin I at rest $)\} /($ angiotensin I at rest $) \times 100(\%)$

Statistics: Data are presented as mean \pm SE. Group comparisons were made by one-way analysis of variance. A probability value $<0.05$ was considered statistically significant.

\section{RESULTS}

Hemodynamics: As shown in Table II, both at rest and peak exercise, heart 
Table II. Hemodynamic Data at Rest and Peak Exercise

\begin{tabular}{|c|c|c|c|c|}
\hline & \multicolumn{2}{|c|}{ Preserved } & \multicolumn{2}{|c|}{ Impaired } \\
\hline & Rest & Peak & Rest & Peak \\
\hline & \multicolumn{2}{|c|}{$*$} & \multicolumn{2}{|c|}{$*$} \\
\hline \multirow[t]{2}{*}{ HR (beat/min) } & $59.3 \pm 8.5$ & $102.7 \pm 16.5$ & $66.5 \pm 4.7$ & $122.0 \pm 23.6$ \\
\hline & \multicolumn{2}{|c|}{$*$} & \multicolumn{2}{|c|}{$*$} \\
\hline \multirow[t]{2}{*}{ Systolic BP (mmHg) } & $152.0 \pm 30.0$ & $180.3 \pm 36.6$ & $125.0 \pm 7.6$ & $178.5 \pm 7.9$ \\
\hline & \multicolumn{4}{|c|}{ N.S. } \\
\hline Work Rate (Watt) & \multicolumn{2}{|c|}{$70.8 \pm 29.2$} & \multicolumn{2}{|c|}{$93.8 \pm 12.5$} \\
\hline
\end{tabular}

$\mathrm{HR}=$ heart rate; $\mathrm{BP}=$ blood pressure. $*, p<0.05$.

Table III. Neurohormonal Factors at Rest and Peak Exercise

\begin{tabular}{|c|c|c|c|c|}
\hline & \multicolumn{2}{|c|}{ Preserved } & \multicolumn{2}{|c|}{ Impaired } \\
\hline & Rest & Peak & Rest & Peak \\
\hline & \multicolumn{2}{|c|}{$*$} & \multicolumn{2}{|c|}{ N.S. } \\
\hline Norepinephrine $(\mathrm{pg} / \mathrm{m} l)$ & $3.8 \pm 9.4$ & $20.8 \pm 20.5$ & $20.2 \pm 33.0$ & $45.8 \pm 41.9$ \\
\hline \multirow[t]{2}{*}{$\operatorname{Renin}(\mathrm{ng} / \mathrm{m} l)$} & $3.6 \pm 3.0$ & $2.0 \pm 1.2$ & $3.9 \pm 2.6$ & $11.1 \pm 16.4$ \\
\hline & $\Gamma$ & \rceil & $\Gamma$ & 7 \\
\hline Aldosterone $(\mathrm{pg} / \mathrm{m} l)$ & $47.2 \pm 29.2$ & $6.7 \pm 3.1$ & $88.2 \pm 56.7$ & $5.2 \pm 3.7$ \\
\hline Angiotensin I $(\mathrm{pg} / \mathrm{m} l)$ & $788 \pm 815$ & $553 \pm 477$ & $838 \pm 563$ & $1620 \pm 1709$ \\
\hline Angiotensin II $(\mathrm{pg} / \mathrm{m} l)$ & $26.0 \pm 13.9$ & $22.5 \pm 13.7$ & $36.6 \pm 24.2$ & $57.2 \pm 42.7$ \\
\hline $\mathrm{BNP}(\mathrm{pg} / \mathrm{m} l)$ & $20.2 \pm 23.4$ & $30.7 \pm 23.2$ & $26.5 \pm 17.5$ & $27.6 \pm 30.2$ \\
\hline
\end{tabular}

Abbreviations; $\mathrm{BNP}=$ brain natriuretic peptide, $\quad *, p<0.05$.

rate tended to be higher in the impaired LV function group, but showed no significant difference compared to that in the preserved LV function group. Heart rate and systolic blood pressure during exercise were significantly $(p<0.05)$ higher than those at rest, in each group, and even in all of the patients, indicating that the ergometer exercise was hard enough to evaluate the neurohormonal factors. The work rate was similar in the two groups.

Neurohormonal factors at baseline and during exercise: There were no significant differences between the two groups in any neurohormonal factors, either at rest or at peak exercise. As shown in Table III, plasma norepinephrine levels were significantly $(p<0.05)$ increased by ergometer exer- 

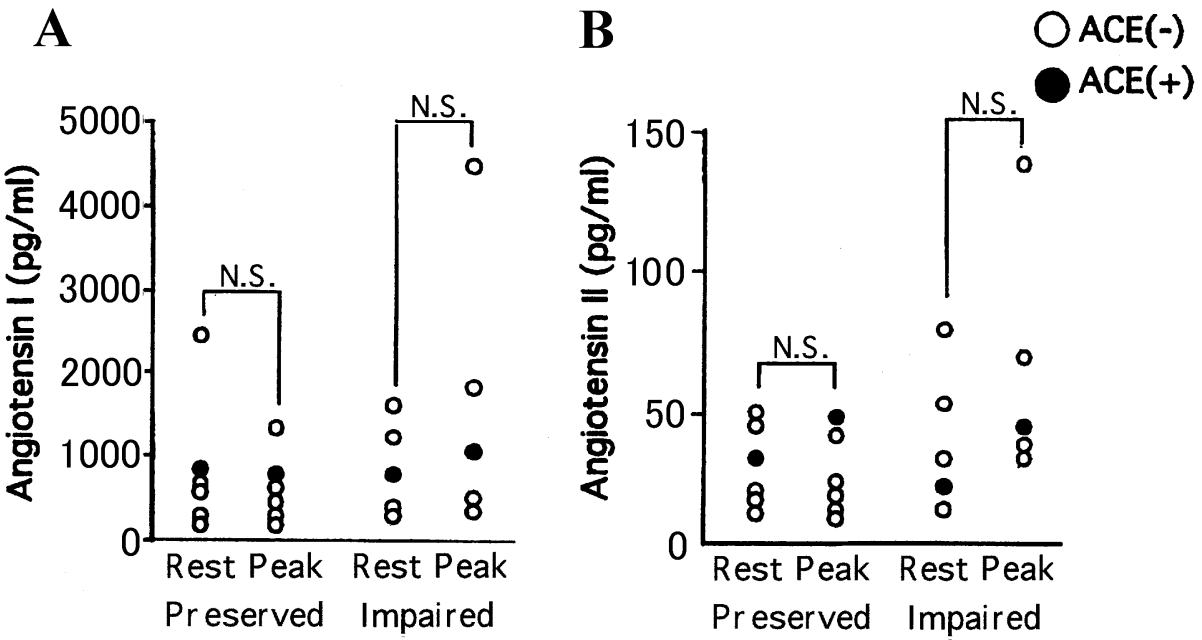

Figure 1. Plasma levels of angiotensin I (A) and II (B) from rest to peak exercise. There was no significant difference in these factors from rest to peak exercise in patients with preserved left ventricular (LV) function. Similar results were seen in patients with impaired LV function.

A

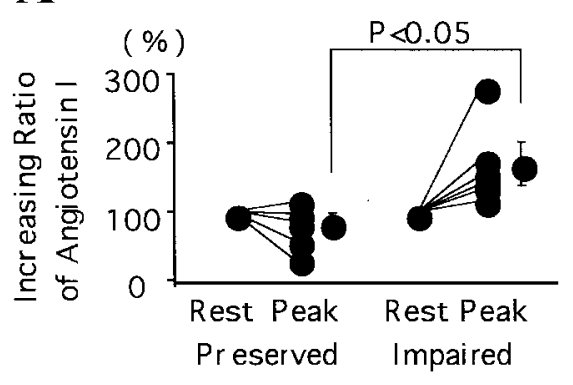

B

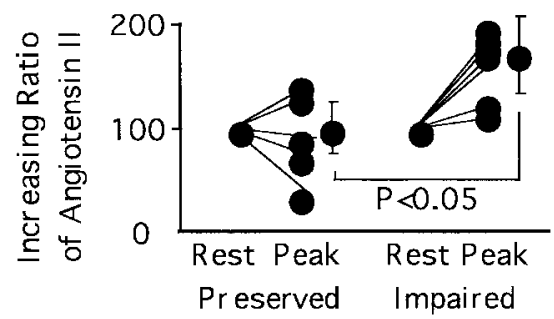

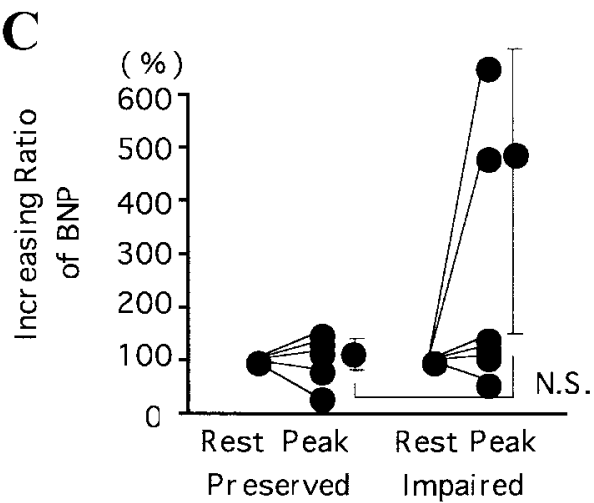

Figure 2. The increasing ratios of plasma angiotensin I, II and brain natriuretic peptide (BNP) from rest to peak exrecise. (A) The increasing ratio of circulating angiotensn I was significantly higher in the group of impaired left ventricular (LV) function than those of preserved LV function. Error bars represent SE. (B) The increasing ratio of plasma angiotensin II from rest to peak exrecise was also significantly higher in patients with impaired LV contraction than those with preserved. Error bars represent SE. (C) There was no significant difference in the increasing ratio of BNP between the two groups. A probability value $<0.05$ was considered statistically significant. Error bars represent SE. 
cise in the preserved LV function group, but not in the impaired group. In both groups, the aldosterone concentration at peak exercise was significantly $(p<0.05)$ decreased compared with that at rest. Each group contains one patient receiving an ACE inhibitor. To examine whether ACE inhibitors affect RAS during exercise, we showed the plasma angiotensin I and II levels in each patient. As shown in Figure 1, patients receiving an ACE inhibitor represent similar plasma levels of angiotensin I and II compared to those of patients without an ACE inhibitor. Although plasma angiotensin I and II levels did not differ between the two groups at rest or at peak exercise (Figure 1), the increasing ratios of angiotensin I and II from rest to peak exercise were significantly $(p<0.05)$ greater in patients with impaired LV function in than those with preserved LV function as shown in Figure 2A and B. Plasma BNP levels, which tended to be higher in patients with preserved LV function at rest, showed no notable change after exercise in the two groups. The increasing ratio of BNP was also evaluated in the same way as angiotensin I and II, which resulted in no significant difference between the two groups (Figure 2C).

\section{DISCUSSION}

The preliminary data demonstrated that the neurohormonal responses to dynamic exercise in patients with IHD are augmented dependent on their LV function by the following two results. First, in patients with severely impaired LV function, the increasing ratios of angiotensin I and II resulted in a greater increase compared to those in patients with preserved LV function. Second, plasma norepinephrine was significantly increased in the group with preserved LV function by supine ergometer exercise, however, it was not altered in the group with impaired LV function. Furthermore, we revealed that the plasma aldosterone levels were decreased after exercise in all of the patients with IHD.

Exercise intolerance is a hallmark of CHF. ${ }^{13)}$ Evaluation of severity of $\mathrm{CHF}$ often requires exercise testing, because baseline measurement of cardiac performance does not correlate well with exercise capacity. ${ }^{13-15)}$ Activated RAAS and the sympathetic nervous system in CHF before exercise $^{2)}$ are believed to contribute to the exercise intolerance in CHF. Furthermore, an angiotensin-converting enzyme inhibitor or beta-adrenergic antagonist improves the prognosis and exercise intolerance in patients with CHF. ${ }^{6,710)}$ The changes in neurohormonal factors to dynamic exercise have not been fully understood in relation to LV function in IHD. In this study, our patients with IHD were divided into two groups according to 
LV ejection fraction, which made it possible to compare the neurohormonal responses in relation to the severity of impaired LV function.

Both at rest and at peak exercise, heart rate tended to be higher in the impaired LV function group, but showed no significant difference compared to that in the preserved LV function group. The work rate was similar in the two groups. These results conflicted with the general prospect, in which resting heart rate is higher and the work rate is lower in the group with impaired LV function. The discrepancy may be due to the number of patients studied. By supine ergometer exercise, our study showed that plasma norepinephrine levels at peak exercise clearly increased in patients with preserved LV function, but not in those with impaired LV function. In the impaired LV function group, although no apparent increase by exercise was seen, the plasma norepinephrine levels both at rest and at peak exercise tended to be higher than those in the preserved LV function group. These observations are consistent with previous studies which showed no difference in peak norepinephrine values at maximal exercise among patients with CHF of varying severity and normal subjects. ${ }^{16,17)}$ Our data are different from previous data with respect to plasma norepinephrine levels. ${ }^{5}$ In the present study, plasma norepinephrine levels were much lower compared to those of Francis, et al., in which the median value was $490 \mathrm{pg} / \mathrm{ml}$. We believe this is due to differences in the patient characteristics observed. Some of our patients had relatively preserved LV systolic function. In contrast, in the VHeft-II study the LVEF of patients was limited to less than $45 \%$. The difference in the severity of LV dysfunction may give rise to the disagreement in the plasma norepinephrine levels.

The aldosterone concentration at peak exercise was significantly decreased compared with that at rest in both groups. Aldosterone secretion is regulated by plasma concentrations of potassium, renin and angiotensin. During exercise, increased plasma renin activity and activated angiotensin stimulate the adrenal cortex to secrete aldosterone. Therefore dynamic exercise should increase aldosterone secretion. However, our result did not confirm to this established theory. The reason is unknown, but we cannot exclude the possibility of drugs, including angiotensin-converting enzyme inhibitors.

The RAAS, which is activated in patients with low cardiac output, operates in concert with the activated adrenergic nervous-adrenal medullary system to maintain arterial pressure. In the SOLVD study, plasma angiotensin II was significantly elevated even in asymptomatic patients and was further elevated in patients with symptomatic heart failure. ${ }^{7)}$ Angiotensin II is a potent peripheral vasoconstrictor and contributes to the 
excessive elevation of systemic vascular resistance. Our results were not consistent with the previous report that showed increased levels of norepinephrine, renin, and angiotensin II. ${ }^{17)}$ The discordance may be due to the following reasons. We divided patients according to their LV systolic function, while the observations of Kato were classified according to NYHA criteria. The etiologic basis of the heart disease is limited to only coronary disease in our study, but that of Kato was heterogeneous. In short, the characteristics of our patients were quite different from those of Kato, et al. In contrast to the previous study, our results demonstrated that not the severity of CHF, but rather LV dysfunction might affect the increased response of angiotensin II. Moreover, plasma angiotensin I was also augmented by dynamic exercise in patients with impaired LV function. The increased angiotensin II may contribute the excessive elevation of systemic vascular resistance and the exercise intolerance in patients with impaired LV function. Thus, increased plasma angiotensin II levels after exercise and its cardiovascular effects are well established in patients with CHF. On the other hand, little is known about plasma angiotensin I. To the best of our knowledge, plasma angiotensin I in cardiovascular disease has never been reported. Since angiotensin I also has some biological effects independent of angiotensin II, ${ }^{18-20)}$ it may act on the cardiovascular system by itself.

Plasma BNP levels are known to be elevated in patients with IHD as well as $\mathrm{CHF}^{21)} \mathrm{BNP}$, which is closely correlated with LV end-diastolic pressure, ${ }^{22)}$ is regarded as a predictor of LV dysfunction at rest. However, we were unable to demonstrate apparent changes in plasma levels of BNP by dynamic exercise in either group. The oscillation of BNP after exercise remains unclear in patients with IHD. Nicholson, et al. reported that the increase in plasma BNP after exercise was at most 1.3-fold, whereas plasma ANP increased 3-fold in patients with IHD, ${ }^{23)}$ suggesting that angiotensin I and II rather than BNP may be relevant predictors for cardiac performance. Most of the clinical trials concerning CHF apply to patients whose LVEF are less than $40 \%$. In practice, it is of significant importance to prevent worsening of CHF even in patients with mild LV dysfunction. Therefore we believe that aggressive therapies using novel predictors for cardiac performance such as BNP, angiotensin I, and II have some advantages in CHF therapy.

We acknowledge that there are several limitations to this study. First the number of patients examined is relatively small. We should add more patients and determine the relationships between exercise and neurohormonal activation at length in future studies. We would like to point out 
that, in such a small number of patients, we could detect differences in the increasing ratio of angiotensin I and II between the two groups, which means they are more sensitive markers for LV dysfunction than other known markers. We should also discuss drug administration. Each group contains one patient receiving an ACE inhibitor. We showed that the effects of ACE inhibitors on plasma levels of angiotensin I and II were minimal, but cannot exclude the possibilities that other drugs such as nitrates or calcium antagonists might affect neurohormonal responses to exercise.

In conclusion, this pilot study has shown that patients with severely impaired LV function had greater increasing ratios in angiotensin I and II from rest to peak exercise compared with patients with mildly impaired LV function. Augmented responses of angiotensin I and II upon dynamic exercise may contribute to the compensatory vasoconstrictory system and exercise intolerance in patients with IHD. The increasing ratios of angiotensin I and II could be valuable markers for LV dysfunction. In terms of clinical importance, we believe that the blockade of angiotensin I and II helps the management of patients with IHD by reducing excess response of the RAAS.

\section{REFERENCES}

1. De Souza MJ, Maresh CM, Maguire MS, Kraemer WJ, Flora GG, Goetz KL. Menstrual status and plasma vasopressin, renin activity, and aldosterone exercise responses. J Appl Physiol 1989; 67: 736-43.

2. Francis GS, Goldsmith SR, Levine TB, Olivari MT, Cohn JN. The neurohumoral axis in congestive heart failure. Ann Intern Med 1984; 101: 370-7.

3. Francis GS, Benedict C, Johnstone DE, et al. Comparison of neuroendocrine activation in patients with left ventricular dysfunction with and without congestive heart failure. A substudy of the Studies of Left Ventricular Dysfunction (SOLVD). Circulation 1990; 82: 1724-9.

4. Meredith IT, Eisenhofer G, Lambert GW, Dewar EM, Jennings GL, Esler MD. Cardiac sympathetic nervous activity in congestive heart failure. Evidence for increased neuronal norepinephrine release and preserved neuronal uptake. Circulation 1993; 88: 136-45.

5. Francis GS, Cohn JN, Johnson G, Rector TS, Goldman S, Simon A. Plasma norepinephrine, plasma renin activity, and congestive heart failure. Relations to survival and the effects of therapy in V-HeFT II. The V-HeFT VA Cooperative Studies Group. Circulation 1993; 87: vI40-8.

6. The CONSENSUS Trial Study Group. Effects of enalapril on mortality in severe congestive heart failure. Results of the Cooperative North Scandinavian Enalapril Survival Study (CONSENSUS). N Engl J Med 1987; 316: 1429-35.

7. The SOLVD Investigators. Effect of enalapril on survival in patients with reduced left ventricular ejection fractions and congestive heart failure. N Engl J Med 1991; 325: 293-302.

8. Cohn JN, Johnson G, Ziesche S, et al. A comparison of enalapril with hydralazine-isosorbide dinitrate in the treatment of chronic congestive heart failure. N Engl J Med 1991; 325: 303-10.

9. Pfeffer MA, Braunwald E, Moye LA, et al. Effect of captopril on mortality and morbidity in patients with left ventricular dysfunction after myocardial infarction. Results of the survival and ventricular enlargement trial. The SAVE Investigators. N Engl J Med 1992; 327: 669-77. 
10. Packer M, Colucci WS, Sackner BJ, et al. Double-blind, placebo-controlled study of the effects of carvedilol in patients with moderate to severe heart failure. The PRECISE Trial. Prospective Randomized Evaluation of Carvedilol on Symptoms and Exercise. Circulation 1996; 94: 2793-9.

11. Kinugawa T, Ogino K, Kitamura H, et al. Catecholamines, renin-angiotensin-aldosterone system, and atrial natriuretic peptide at rest and during submaximal exercise in patients with congestive heart failure. Am J Med Sci 1996; 312: 110-7.

12. Ueyama M, Nishimura H, Kubota J, Kawamura K. Hemodynamic and neurohumoral responses to exercise in patients with congestive heart failure. Jpn Circ J 1993; 57: 411-7.

13. Franciosa JA. Exercise testing in chronic congestive heart failure. Am J Cardiol 1984; 53: 1447-50.

14. Franciosa JA, Ziesche S, Wilen M. Functional capacity of patients with chronic left ventricular failure. Relationship of bicycle exercise performance to clinical and hemodynamic characterization. Am J Med 1979; 67: 460-6.

15. Franciosa JA, Park M, Levine TB. Lack of correlation between exercise capacity and indexes of resting left ventricular performance in heart failure. Am J Cardiol 1981; 47: 33-9.

16. Colucci WS, Ribeiro JP, Rocco MB, et al. Impaired chronotropic response to exercise in patients with congestive heart failure. Role of postsynaptic beta-adrenergic desensitization. Circulation 1989; 80: 314-23.

17. Kato M, Kinugawa T, Omodani H, et al. Responses of plasma norepinephrine and renin-angiotensin-aldosterone system to dynamic exercise in patients with congestive heart failure. J Card Fail 1996; 2: 103-10.

18. Peach MJ, Bumpus FM, Khairallah PA. Release of adrenal catecholamines by angiotensin I. J Pharmacol Exp Ther 1971; 176: 366-76.

19. Peach MJ. Adrenal medullary stimulation induced by angiotensin I, angiotensin II, and analogues. Circ Res 1971; 28: 107-17.

20. Xu ZH, Shimakura K, Yamamoto T, Wang LM, Mineshita S. Pulmonary edema induced by angiotensin I in rats. Jpn J Pharmacol 1998; 76: 51-6.

21. Marumoto K, Hamada M, Aburaya M, Hiwada K. Augmented secretion of atrial and brain natriuretic peptides during dynamic exercise in patients with old myocardial infarction. Jpn Circ J 1995; 59: 715-24.

22. Maeda K, Tsutamoto T, Wada A, Hisanaga T, Kinoshita M. Plasma brain natriuretic peptide as a biochemical marker of high left ventricular end-diastolic pressure in patients with symptomatic left ventricular dysfunction. Am Heart J 1998; 135: 825-32.

23. Nicholson S, Richards M, Espiner E, Nicholls G, Yandle T. Atrial and brain natriuretic peptide response to exercise in patients with ischaemic heart disease. Clin Exp Pharmacol Physiol 1993; 20: 535-40 
